\title{
Tetanus Toxoid Helper Peptide
}

National Cancer Institute

\section{Source}

National Cancer Institute. Tetanus Toxoid Helper Peptide. NCI Thesaurus. Code C38134.

Obtained by genetic engineering from the bacterial Clostridium tetani toxoid, tetanus toxoid helper peptide QYIKANSKFIGITEL (amino acids 830-844) binds to class II MHC molecules as a nonspecific vaccine helper epitope (adjuvant) and induces an increased (and long term) immune response by increasing the helper T-cell response. ( $\mathrm{NCI05)}$ 